\title{
Pérdida auditiva inducida por ruido en estudiantes de la carrera de odontología
}

\author{
Noise induced hearing loss in dentistry students
}

Eduardo Fuentes $\mathbf{L}^{1,2}$, Consuelo Rubio $\mathbf{M}^{3}$, Felipe Cardemil $\mathbf{M}^{1,4}$.

\begin{abstract}
RESUMEN
Introducción: Los odontólogos se exponen desde su formación universitaria a sonidos potencialmente dañinos en forma rutinaria.

objetivo: Determinar si la presencia de escotoma en las frecuencias 3, 4 y $6 \mathrm{kHz}$ está asociada a la exposición a ruido de implementos dentales.

Material y método: Se realizó un estudio de corte transversal en estudiantes expuestos a ruido en sus actividades prácticas dentales y otro grupo no expuesto. Se realizaron evaluaciones auditivas a ambos grupos, además de una sonometría y dosimetría en el laboratorio dental.

Resultados: Se incluyeron 50 estudiantes de odontología expuestos a ruido de implementos dentales y 107 de otras carreras no expuestos. No se observaron diferencias significativas al comparar los umbrales de ambos grupos. Sin embargo, la frecuencia de escotoma en $4 \mathrm{kHz}$ como en $6 \mathrm{kHz}$ del oído izquierdo fue significativamente mayor en el grupo expuesto. Finalmente, la posibilidad de presentar un escotoma en 4 y $6 \mathrm{kHz}$ del oído izquierdo estuvo significativamente asociada a exposición a ruido dental.
\end{abstract}

Conclusión: La exposición a ruido derivado de prácticas dentales está asociado a escotoma en las frecuencias agudas. Sin embargo, la gran mayoría de los sujetos expuestos no evidenció una elevación de los umbrales por sobre el límite considerado normal.

Palabras clave: Odontólogos, pérdida auditiva inducida por ruido, estudiantes universitarios.

1 Programa de Doctorado en Salud Pública, Escuela de Salud Pública, Universidad de Chile.

2 Facultad de Ciencias de la Salud, Escuela de Fonoaudiología, Universidad San Sebastián.

3 Facultad de Odontología, Universidad San Sebastián.

4 Servicio de Otorrinolaringología, Hospital Barros Luco Trudeau, Universidad de Chile. 


\section{ABSTRACT}

Introduction: Starting with their time at college, dentists are routinely exposed to potentially harmful sounds.

AIM: To determine whether the presence of notch in 3, 4 and $6 \mathrm{kHz}$ frequencies is associated with exposure to noise from dental tools.

Material and method: A cross-sectional study was performed with a group of students exposed to noise in their dental practices and with another group that was not exposed. Hearing tests were performed with both groups, along with a sound measurement and dosimetry in the dental laboratory.

Results: Fifty dental students were exposed to the noise of dental implements and 107 students were not exposed. No significant differences were observed when comparing the thresholds of both groups. However, the frequency of notch at $4 \mathrm{kHz}$, as at $6 \mathrm{kHz}$ in the left ear, was significantly higher in the exposed group.

Conclusion: Noise exposure derived from dental practices is associated with notch at high frequencies. However, the vast majority of the subjects showed no evidence of a threshold higher than the limit that is considered normal.

Key words: Dentists, noise-induced hearing loss, college students.

\section{INTRODUCCIÓN}

Existen profesionales que en su práctica clínica se exponen a sonidos potencialmente dañinos, como los odontólogos, los que se ven inmersos en ambientes con elevado ruido generado por el instrumental rotatorio utilizado en su práctica profesional (turbina, micromotor, pieza de mano). A lo largo del tiempo los estudios en el área han mostrado resultados dispares. Se ha señalado que estos profesionales presentarían pérdida auditiva en el rango de las altas frecuencias ${ }^{1-4}$, también se ha indicado que si bien existe dicho riesgo, éste sería mínimo ya que el nuevo equipamiento es menos ruidos $0^{5}$. Por otro lado, se ha reportado que los dentistas no presentarían pérdida auditiva asociada a la exposición a ruido $0^{6-8}$. Estos últimos reportes probablemente se explican dado el reducido tamaño muestral considerado y/o a los criterios utilizados para definir la presencia de pérdida auditiva por ruido. Además, se contradicen con el hecho de que las turbinas dentales pueden alcanzar los $110 \mathrm{~dB}$, lo que es potencialmente dañino para el sistema auditivo ${ }^{9}$. En relación al espectro frecuencial de dichos elementos, no sólo se han reportado emisiones elevadas en el rango audible (alrededor de 6 a $7 \mathrm{kHz}$ ) ${ }^{10}$ sino que adicionalmente se generan picos en los ultrasonidos (sobre $20 \mathrm{kHz})^{11,12}$.

Es posible afirmar que los odontólogos se ven inmersos en ambientes ruidosos desde su formación universitaria. Al comparar los umbrales auditivos de alumnos de primer año de la carrera con mediciones posteriores a 5 años de experiencia laboral, se evidenció un marcado descenso en los 4 kHz . También se han observado cambios en la amplitud de las emisiones otoacústicas para las frecuencias 4 y $6 \mathrm{kHz}$ del oído izquierdo y $6 \mathrm{kHz}$ del oído derecho al comparar las mediciones previas y posteriores a una jornada de actividad práctica dental de los estudiantes ${ }^{13}$. En las clases prácticas dentales, el ambiente acústico se caracteriza por altos niveles de ruido en relación con otras carreras, debido a que el sonido producido por el instrumental dental se ve potenciado por el uso de varios equipos al mismo tiempo ${ }^{14}$.

Resulta de interés estudiar el estado del sistema auditivo de los jóvenes estudiantes de la carrera de odontología, quienes están inmersos en un ambiente con elevados niveles de ruido en sus actividades prácticas desde muy temprana edad.

\section{OBJETIVO}

El objetivo del presente estudio fue determinar si la existencia de una alteración en los umbrales audiométricos, conformando un escotoma en las frecuencias agudas, está asociada a la exposición a ruido de los implementos dentales.

\section{MATERIAL Y MÉTODO}

Se realizó un estudio de corte transversal durante el año 2012 en el que se invitó a participar a 
estudiantes universitarios de una universidad de la región Metropolitana. Inicialmente, se conformó un grupo de alumnos voluntarios de la carrera de odontología y otro de estudiantes pertenecientes a otra carrera del área de ciencias de la salud. A través de historia clínica, exploración mediante otoscopía y examen audiométrico e impedanciométrico se excluyeron a todos aquellos estudiantes que presentaron patologías de oído no relacionadas a pérdida auditiva inducida por ruido; en su mayoría tapones de cerumen ( $n=14 ; 3$ en el caso de los estudiantes de odontología y 11 para los estudiantes de otras carreras). En cuanto a las evaluaciones audiométricas se efectuaron en cabina silente (sonoaislada y sonoamortiguada) con un audiómetro Interacoustics AC40 calibrado según norma ANSI S3.6. Con el objetivo de excluir los cambios transitorios de umbral sólo se realizaron los exámenes audiométricos a aquellos estudiantes que indicaron no exponerse a ruido las últimas 24 horas previas, sea este ruido de carácter recreacional (uso de reproductores de archivos mp3, asistencia a conciertos, etc.) o producido por los aparatos del laboratorio de prácticas dentales. En el caso de los alumnos de odontología se excluyeron a aquellos que aún no asistían a actividades prácticas que implicaban el uso de rotomotores, es decir a los alumnos de $1^{0}$ y $2^{\circ}$ año $(n=20)$. En el caso de los estudiantes de otras carreras del área de la salud, y con el objetivo de parear la muestra en cuanto a edad, también se excluyeron a los estudiantes que aún no cursaban el $3^{\circ}$ año $(n=124)$. Cada estudiante fue informado de la realización del estudio y aceptó participar en éste.

La variable desenlace fue la presencia de alteraciones en los umbrales auditivos en la forma de escotoma (notch) en las frecuencias agudas $3 \mathrm{kHz}, 4$ $\mathrm{kHz}$ y $6 \mathrm{kHz}$. Como en la presente investigación se utilizó un audiómetro con auriculares "Telephonics TDH-39" se realizó una corrección de 6 dB en el umbral obtenido en la frecuencia $6 \mathrm{kHz}$, para evitar la presencia de un descenso audiométrico de causa artificial $^{15}$. Se determinó la presencia de escotoma tomando en cuenta el criterio propuesto por Coles y $\mathrm{col}^{16}$, en donde el nivel del umbral de audición (en $\mathrm{dB} H T L$ ) en $3 \mathrm{y} / 04 \mathrm{y} / 06 \mathrm{kHz}$ debe ser al menos 10 $\mathrm{dB}$ superior a $1 \mathrm{kHz} 02 \mathrm{kHz}$ y $8 \mathrm{kHz}$.

Como variable predictora se incluyó la exposición a ruido en las actividades prácticas denta- les. Dicha variable se trató en forma dicotómica, asumiendo la existencia de un grupo expuesto a ruido dental y otro no expuesto. Dicha asunción se sustenta además en que se constató que ninguno de los estudiantes del grupo expuesto utilizó algún tipo de protección auditiva al momento de asistir a las prácticas dentales. Con el objetivo de cuantificar la exposición a ruido en las prácticas dentales se realizaron mediciones en terreno utilizando un sonómetro (marca CEL, modelo 440). Se obtiene el nivel continuo equivalente (Leq) de la jornada de clases, el espectro de los niveles máximos equivalentes y la dosis de ruido proyectada. El registro fue realizado en dos sesiones típicas de laboratorio con una duración de tres horas cada una.

Otra variable considerada fue el sexo de los estudiantes ya que se ha reportado que la susceptibilidad al ruido puede ser influenciada por éste. Tanto Berger y $\mathrm{col}^{17}$ quienes estudiaron trabajadores en industrias, como Niskar y $\mathrm{col}^{18}$ los que consideraron sujetos entre 6 y 19 años, observaron una menor frecuencia de pérdida producto del ruido en el sexo femenino. Además, se cree que podría existir una exposición diferencial según sexo a actividades ruidosas ${ }^{19,20}$.

Para el análisis estadístico de los datos obtenidos en la presente investigación se utilizó el programa STATA versión 12. Las variables cuantitativas fueron descritas mediante promedio $\mathrm{y}$ desviación estándar o mediana y rango mínimo y máximo, según correspondiera. En el caso de la variables categóricas se reportaron las frecuencias absolutas y relativas. Se constató a través de la prueba de Shapiro-Wilk que la variable "umbrales audiométricos" no poseía una distribución normal, por lo que se utilizó la prueba no paramétrica de Mann Whitney para comparar los resultados entre expuestos y no expuestos. Una vez categorizada la anterior variable en base a la definición de escotoma propuesta por Coles y col $^{16}$ se procedió a determinar la prevalencia en las frecuencias 3,4 y $6 \mathrm{kHz}$. Se utilizó un método binomial exacto para el cálculo de los intervalos de confianza. Finalmente, se crearon modelos de regresión logística estimando los odds ratio, con sus respectivos intervalos de confianza. Cada modelo tuvo como variable respuesta la presencia de escotoma en las frecuencias 3,4 y $6 \mathrm{kHz}$. Dada la asimetría de los grupos en relación a la composición según sexo, se procedió 
a crear modelos de regresión logística múltiple, donde se controló este factor de confusión, ajustando por dicha variable. Se consideró un nivel de significancia del $5 \%$ para todos los contrastes de hipótesis realizados.

\section{RESULTADOS}

El grupo de sujetos expuestos quedó conformado por 50 estudiantes de la carrera de odontología que poseían al menos un semestre de exposición a ruido emanado por los instrumentos de trabajo dental, y el grupo no expuesto por 107 estudiantes pertenecientes a otras carreras del área de la salud. En la muestra destacó que el grupo de estudiantes expuestos presentó una proporción mayor de hombres en comparación al no expuesto. Sin embargo, la edad promedio de ambos grupos fue similar. En la Tabla 1 se muestran las características de la muestra considerada.

En cuanto a los umbrales audiométricos, las medianas en todas las frecuencias no superaron el límite de $20 \mathrm{~dB}$ considerado como normal. No se observaron diferencias estadísticamente significativas al comparar los umbrales de ambos grupos tanto para el oído derecho como para el izquierdo (Tabla 2).

En la Tabla 2 destaca que existen valores máximos fuera del rango normal, en las frecuencias por sobre los $2 \mathrm{kHz}$. A través de la obtención del percentil 90 se determinó que dichos valores corresponden a outliers. En la frecuencia $4 \mathrm{kHz}$ del oído izquierdo dos de estos valores extremos los presentaron sujetos no expuestos y dos los expuestos. En el caso de estos últimos, son los que presentan una mayor elevación del umbral. En el oído derecho de la mencionada frecuencia sólo existía un sujeto con una pequeña desviación de $5 \mathrm{~dB}$ con respecto al nivel considerado normal, el que formaba parte del grupo no expuesto. En la frecuencia $6 \mathrm{kHz}$ del oído izquierdo los valores extremos los aportaron tanto expuestos como no expuestos en igual medida ( 2 casos cada uno), sin embargo, la mayor desviación con respecto a la normalidad se observó en un sujeto del grupo expuesto. En el caso del oído derecho dos sujetos presentaron pequeñas elevaciones de $5 \mathrm{~dB}$ (grupo expuesto y no expuesto) y uno $15 \mathrm{~dB}$ (no expuesto).

En base a la definición operacional de escotoma mencionada previamente16, se estimó la prevalencia y razón de prevalencia con sus respectivos intervalos de confianza exactos para las frecuencias 3,4 y $6 \mathrm{kHz}$ en ambos grupos y oídos (Tablas 3 y 4). En el caso del oído izquierdo la frecuencia de escotoma en $4 \mathrm{kHz}$ fue 3,6 veces mayor en el grupo de sujetos expuestos en relación a los no expuestos. Debido a que los intervalos de confianza no incluyeron el valor nulo, la asociación fue estadísticamente significativa. Algo similar ocurrió en 6 $\mathrm{kHz}$ donde la frecuencia de escotoma es 1,8 veces mayor en los expuestos que en los no expuestos, también siendo estadísticamente significativa. En el oído derecho no se observaron razones de prevalencia estadísticamente significativas.

Tabla 1. Características de un grupo de estudiantes universitarios de odontología y de otras carreras del área de la salud

\begin{tabular}{|l|c|c|c|}
\hline Variable & Grupo expuesto $(\mathrm{n}=50)$ & Grupo no expuesto $(\mathrm{n}=107)$ & Valor de $p$ \\
\hline Edad & 22 & 22 & \\
\hline Mediana & $20-30$ & $20-30$ & $0,327^{\text {a }}$ \\
Mínimo - Máximo & & & $11 /(89,7 \%)$ \\
\hline Sexo (\%) & $22(44 \%)$ & $96 /(10,3 \%)$ & $<0,001^{\mathrm{b}}$ \\
\hline Masculino & $28(56 \%)$ & & \\
\hline
\end{tabular}

a Mann-Whitney.

${ }^{\mathrm{b}}$ Chi-cuadrado. 
Tabla 2. Umbrales audiométricos de ambos oídos en un grupo de estudiantes universitarios de odontología y de otras carreras del área de la salud

\begin{tabular}{|c|c|c|c|c|c|c|}
\hline \multirow[t]{2}{*}{ Frecuencia } & \multicolumn{3}{|c|}{ Oído derecho en decibeles } & \multicolumn{3}{|c|}{ Oído izquierdo en decibeles } \\
\hline & Mediana & Min - Max & Valor $p$ & Mediana & $\operatorname{Min}-\operatorname{Max}$ & Valor de $p$ \\
\hline \multicolumn{7}{|l|}{$1 \mathrm{kHz}$} \\
\hline Grupo expuesto & 5 & $0-15$ & & 5 & $0-20$ & \\
\hline Grupo no expuesto & 5 & $0-20$ & 0,29 & 5 & $0-20$ & 0,41 \\
\hline \multicolumn{7}{|l|}{$2 \mathrm{kHz}$} \\
\hline Grupo expuesto & 5 & $0-20$ & & 5 & $0-20$ & \\
\hline Grupo no expuesto & 5 & $0-25$ & 0,98 & 5 & $0-20$ & 0,58 \\
\hline \multicolumn{7}{|l|}{$3 \mathrm{kHz}$} \\
\hline Grupo expuesto & 0 & $0-15$ & & 0 & $0-35$ & \\
\hline Grupo no expuesto & 5 & $0-50$ & 0,65 & 5 & $0-30$ & 0,93 \\
\hline \multicolumn{7}{|l|}{$4 \mathrm{kHz}$} \\
\hline Grupo expuesto & 5 & $0-20$ & & 5 & $0-65$ & \\
\hline Grupo no expuesto & 5 & $0-25$ & 0,97 & 5 & $0-25$ & 0,33 \\
\hline \multicolumn{7}{|l|}{$6 \mathrm{kHz}$} \\
\hline Grupo expuesto & 4 & $0-24$ & & 4 & $0-49$ & \\
\hline Grupo no expuesto & 4 & $0-34$ & 0,41 & 4 & $0-29$ & 0,46 \\
\hline \multicolumn{7}{|l|}{$8 \mathrm{kHz}$} \\
\hline Grupo expuesto & 5 & $0-15$ & & 5 & $0-15$ & \\
\hline Grupo no expuesto & 5 & $0-30$ & 0,78 & 5 & $0-30$ & 0,20 \\
\hline
\end{tabular}

Tabla 3. Prevalencia y razones de prevalencia (RP) de escotoma en el oído izquierdo en un grupo de estudiantes universitarios de odontología y de otras carreras del área de la salud

\begin{tabular}{|lcccc|}
\hline Frecuencia & $\begin{array}{c}\text { No expuestos } \\
\text { (IC al 95\%) }\end{array}$ & $\begin{array}{c}\text { Expuestos } \\
\text { (IC al 95\%) }\end{array}$ & $\begin{array}{c}\text { Razón prevalencia (RP) } \\
\text { (IC al 95\%) }\end{array}$ & Valor de $\mathrm{p}$ \\
\hline $3 \mathrm{kHz}$ & 1,9 & 8,0 & 4,3 & 0,082 \\
$4 \mathrm{kHz}$ & $(0,0-6,6)$ & $(2,2-19,2)$ & $(0,8-22,6)$ & \\
& 5,6 & 2,0 & 0,4 & 0,432 \\
$6 \mathrm{kHz}$ & $(2,0-11,8)$ & $(0,0-10,6)$ & $(0,0-2,9)$ & \\
& 2,8 & 6,0 & 2,1 & 0,384 \\
\hline
\end{tabular}

Tabla 4. Prevalencia y razones de prevalencia (RP) de escotoma en el oído derecho en un grupo de estudiantes universitarios de odontología y de otras carreras del área de la salud

\begin{tabular}{|lcccc|}
\hline Frecuencia & $\begin{array}{c}\text { No expuestos } \\
\text { (IC al 95\%) }\end{array}$ & $\begin{array}{c}\text { Expuestos } \\
\text { (IC al 95\%) }\end{array}$ & $\begin{array}{c}\text { Razón prevalencia (RP) } \\
\text { (IC al 95\%) }\end{array}$ & Valor de $p$ \\
\hline $3 \mathrm{kHz}$ & 1,9 & 8,0 & 4,3 & 0,082 \\
$4 \mathrm{kHz}$ & $(0,0-6,6)$ & $(2,2-19,2)$ & $(0,8-22,6)$ & \\
& 5,6 & 2,0 & 0,4 & 0,432 \\
$6 \mathrm{kHz}$ & $(2,0-11,8)$ & $(0,0-10,6)$ & $(0,0-2,9)$ & \\
& 2,8 & 6,0 & 2,1 & 0,384 \\
\hline
\end{tabular}


En cuanto a los resultados de los modelos creados a través de regresión logística, cuya variable respuesta fue la presencia de escotoma en las frecuencias agudas, destacó que la posibilidad de presentar escotoma en la frecuencia $4 \mathrm{kHz}$ del oído izquierdo fue 3,7 veces mayor en el caso de los expuestos a ruido en actividades practicas dentales; siendo esto estadísticamente significativo. Similarmente, la posibilidad de presentar escotoma en la frecuencia $6 \mathrm{kHz}$ del mismo oído es 2,3 veces mayor en el caso de los expuestos a ruido dental que en los no expuestos; siendo esto estadísticamente significativo. Todos los valores reportados son ajustados de acuerdo al sexo del estudiante debido a que hubo una diferencia en la proporción entre los grupos, y a la importancia teórica de esa covariable ${ }^{17-20}$ (Tabla 5).

Finalmente, en cuanto a las mediciones obtenidas con sonómetro se obtuvo que la frecuencia con mayor nivel emitida por el rotomotor correspondió a $4 \mathrm{kHz}$ (mediana de 68,1 dB, con un mínimo de 67,5 y un máximo de 69,4 dB). Sin embargo, el ruido registrado por el sonómetro ubicado al centro del laboratorio mostró ser mayor para las frecuencias 0,5 y $0,25 \mathrm{kHz}$; alcanzando un nivel de presión sonora equivalente ponderado en curva $A$ (LeqA) de 63,0 y 60,6 dB, respectivamente. De acuerdo a lo anterior, la dosis proyectada a 8 horas de trabajo continuo sería de 3,1\%.

\section{DISCUSIÓN}

Se evaluó auditivamente a 50 jóvenes estudiantes que pertenecían a la carrera de odontología y 107 a otras carreras del área de la salud. En el caso del grupo de estudiantes de odontología, éstos se encontraban expuestos a ruido del instrumental dental. Los umbrales auditivos obtenidos fueron corregidos considerando que el audiómetro contaba con auriculares que artificialmente podrían elevarlos. A su vez, se objetivó mediante sonometría el nivel y frecuencia del ruido presente en los laboratorios de actividades prácticas dentales.

En cuanto a las diferencias en los umbrales auditivos del total de la muestra, éstas no fueron significativas. Sólo destacó la presencia de sujetos con elevación del umbral en las frecuencias 4 y 6 $\mathrm{kHz}$ donde los que evidenciaron una mayor pérdida pertenecían al grupo de sujetos expuestos. No todos los expuestos a ruido presentan finalmente una pérdida auditiva, se ha observado que existe una gran variabilidad en cuanto a la susceptibilidad entre los individuos ${ }^{21,22}$. Además, se ha reportado una asociación entre algunas variantes genéticas específicas y la prevalencia y severidad de pérdida auditiva inducida por ruido en trabajadores expuestos a éste ${ }^{23,24}$. En la presente investigación es probable que para aquellos sujetos que mostraron una mayor elevación de los umbrales, ésta sea explicada en parte por la susceptibilidad derivada de factores personales, dada la temprana edad en que se manifestó la pérdida auditiva.

En cuanto a la frecuencia de escotoma, ésta fue significativamente mayor en el grupo de sujetos expuestos para las frecuencias 4 y $6 \mathrm{kHz}$ del oído izquierdo, lo que es similar a lo observado en estudios previos ${ }^{1,9,13}$. Esto último es concordante con las mediciones realizadas con sonómetro al instrumental dental (turbina, micromotor, pieza de mano) utilizado por los estudiantes; donde la frecuencia $4 \mathrm{kHz}$ presentó los mayores picos. En cuanto a la asimetría del daño, la razón podría estar relacionada con la menor distancia entre el oído izquier-

Tabla 5. Odds ratios de exposición a ruido ajustados por sexo en un grupo de estudiantes universitarios de odontología y de otras carreras del área de la salud

\begin{tabular}{|c|c|c|c|c|c|c|}
\hline Variables & $\begin{array}{c}\text { Derecho } \\
3 \mathrm{kHz} \\
(\mathrm{IC} \mathrm{al} \mathrm{95 \% )}\end{array}$ & $\begin{array}{c}\text { Izquierdo } \\
3 \mathrm{kHz} \\
\text { (IC al 95\%) }\end{array}$ & $\begin{array}{c}\text { Derecho } \\
4 \mathrm{kHz} \\
\text { (IC al 95\%) }\end{array}$ & $\begin{array}{c}\text { Izquierdo } \\
4 \mathrm{kHz} \\
\text { (IC al 95\%) }\end{array}$ & $\begin{array}{c}\text { Derecho } \\
6 \mathrm{kHz} \\
(\mathrm{IC} \mathrm{al} \mathrm{95 \% )}\end{array}$ & $\begin{array}{c}\text { Izquierdo } \\
6 \mathrm{kHz} \\
(\mathrm{IC} \text { al } 95 \%)\end{array}$ \\
\hline \multicolumn{7}{|c|}{ Exposición a ruido } \\
\hline Expuestos & $\begin{array}{c}4,52 \\
(0,71-28,62)\end{array}$ & $\begin{array}{c}1,24 \\
(0,25-6,17)\end{array}$ & $\begin{array}{c}0,35 \\
(0,04-3,41)\end{array}$ & $\begin{array}{c}3,69^{*} \\
(1,15-11,77)\end{array}$ & $\begin{array}{c}1,94 \\
(0,32-11,6)\end{array}$ & $\begin{array}{c}2,32^{*} \\
(1,03-5,22)\end{array}$ \\
\hline \multicolumn{7}{|l|}{ Sexo } \\
\hline Masculino & $\begin{array}{c}1,03 \\
(0,16-6,72)\end{array}$ & $\begin{array}{c}1,15 \\
(0,19-6,96)\end{array}$ & $\begin{array}{c}0,92 \\
(0,09-9,13)\end{array}$ & $\begin{array}{c}1,46 \\
(0,44-4,87)\end{array}$ & $\begin{array}{c}1,44 \\
(0,21-9,77)\end{array}$ & $\begin{array}{c}0,98 \\
(0,39-2,51)\end{array}$ \\
\hline
\end{tabular}

${ }^{*} p<0,05$. 
do y los rotomotores en el caso de los sujetos diestros, y que la cabeza actúa como sombra atenuando entre 10-15 dB las frecuencias agudas ${ }^{9,13}$.

Es necesario considerar que a pesar de observar diferencias en las prevalencias de escotoma en ambos grupos, esto no necesariamente constituyó una elevación por el límite de lo considerado normal (20 dB). Esto se debe a que fue utilizado el criterio propuesto por Coles y coll ${ }^{16}$ donde con al menos $10 \mathrm{~dB}$ de diferencia entre cierta frecuencia y sus vecinas es posible señalar la existencia de escotoma; se estaría identificando un estadio temprano de pérdida auditiva inducida por ruido.

Otro aspecto importante es que en el ruido medido en el laboratorio dental resaltó la energía presente en las frecuencias graves $(0,25-0,5 \mathrm{kHz})$. Si bien éstas no alteran mayormente la capacidad auditiva, se ha observado que a niveles moderados (50 dBA, o decibeles ponderados según audición humana) podrían afectar negativamente las funciones visuales, concentración y la atención selectiva y continua, especialmente en aquellas personas más sensibles a este tipo de sonidos ${ }^{25}$. Futuras investigaciones deberían considerar el evaluar otros efectos no auditivos de la exposición a ruido, como los mencionados anteriormente y de esta forma abordar el tema en su globalidad.

Finalmente, es necesario considerar el gran número de mujeres que presentó el grupo no expuesto a ruido en prácticas dentales, lo cual es concordante con lo observado en la composición de algunas carreras del área de la salud como Fonoaudiología, Nutrición y Dietética, Terapia Ocupacional, etc. Esta distribución no se mantuvo en el caso de los alumnos de odontología (expuestos a ruido) ya que ambos sexos mostraron proporciones relativamente similares. Como se mencionó previamente, debido a esto es que se incluyó el sexo como variable confusora en los modelos de regresión logística múltiple (ajustándolos por dicha variable). Pese a lo anterior, la imposibilidad de ajustar por sexo al momento de calcular las razones de prevalencia, podría en cierta forma estar sobreestimando el efecto de la exposición.

Estos resultados podrían fundamentar el considerar la mejora en las condiciones acústicas de los laboratorios dentales y/o el suministrar protección auditiva a los alumnos, con el propósito de disminuir la exposición temprana a ruido ya que se ha reportado que aquellos oídos expuestos a temprana edad no "envejecen" en la misma medida que los no expuestos, presentando un ritmo mayor en cuanto al descenso en los umbrales audiométricos ${ }^{26-29}$.

\section{CONCLUSIONES}

En conclusión, la frecuencia de los escotomas en 4 y 6 $\mathrm{kHz}$ del oído izquierdo fue significativamente mayor en el grupo de estudiantes expuestos a ruido producido por el instrumental odontológico en comparación a los no expuestos. A su vez, dicha exposición mostró estar asociada significativamente a la presencia de escotoma en las frecuencias anteriormente mencionadas. La asimetría del daño podría ser explicada por la forma en que es utilizado el instrumental dental según lateralidad del odontólogo y la posición que adopta éste al atender al paciente. A pesar de la mayor frecuencia de escotomas en el grupo de expuestos, la mayoría de los sujetos no evidenció una elevación de los umbrales por sobre el límite considerado normal. Lo anterior podría ser el reflejo de un daño reciente. Finalmente, destacó la gran cantidad de energía acústica presente en las frecuencias graves, lo que pondría de manifiesto la existencia de condiciones acústicas desfavorables en los laboratorios de actividades prácticas dentales evaluados.

\section{Agradecimientos}

Este estudio contó con financiamiento del fondo concursable de la Universidad San Sebastián (Proyecto $\left.\mathrm{N}^{0}: 2012-0001-\mathrm{I}\right)$. Los autores declaran no presentar conflictos de interés en la realización del presente estudio.

\section{BIBLIOGRAFÍA}

1. Zubick HH, Tolentino AT, Boffa J. Hearing loss and the high speed dental handpiece. Am J Public Health 1980; 70: 633-5.

2. Setcos JC, Mahyuddin A. Noise levels encountered in dental clinical and laboratory practice. Int $J$ Prosthodont 1998; 11: 150-7.

3. Coles, RRA, Hoare NW. Noise induced hearing loss and the dentist. Br Dent J 1985; 159: 209-18.

4. Tayler W, Pearson J, Mair A. The hearing threshold levels of dental practitioners exposed to air turbine drill noise. Br Dent J 1965; 118: 206-9. 
5. Wilson C, Vaidyanathan T, Cinotti W, Cohen S, Wang $S$. Hearing-damage risk and communication interference in dental practice. J Dent Res 1990; 69: 489-93.

6. Rahкo A, Karma P, Rahko K, Kataja M. High frequency hearing of dental personnel. Community Dent Oral Epidemiol 1988; 16: 268-70.

7. Forman-Franco B, Abramson AL, Stein T. High-speed drill noise and hearing: audiometric survey of 70 dentists. J Am Dent Assoc 1978; 97: 479-82.

8. Man A, Neuman H, Assif D. Effect of turbine dental drill noise on dentists' hearing. Isr J Med Sci 1982; 18: 475-7.

9. GiJbels et AL. Potential occupational health problems for dentists in Flanders, Belgium. Clin Oral Invest Flanders 2006; 10: 8-16.

10. Altinoz H, Gokbudak R, Bayraktar A, Belli S. A pilot study of measurement of the frequency of sounds emitted by high-speed dental air turbines. J Oral Sci 2001; 43: 189-92.

11. Barek S, Adam 0, Motsch J. Large band spectral analysis and harmful risks of dental turbines. Clin Oral Investig 1999; 3: 49-54.

12. Sorainen E, Rrtkönen E. Noise level and ultrasound spectra during burring. Clin Oral Investig 2002; 6: 133-6.

13. Bali N, Acharya S, Anup N. An assessment of the effect of sound produced in a dental clinic on the hearing of dentists. Oral Health Prev Dent 2007; 5: 187-91.

14. Sampaio Fernandes J, Carvalho A, Gallas M, Vaz P, Matos P. Noise levels in dental schools. Eur J Dent Educ 2006; 28:32-7.

15. OseI-LAH V, YEOH LH. High frequency audiometric notch: An outpatient clinic survey. International Journal of Audiology 2010; 49: 95-8.

16. Coles R, Lutman ME, \& Buffin J. Guidelines on the diagnosis of noise-induced hearing loss for medicolegal purposes. Clin Otolaryngol 2000; 25: 264-73.

17. Berger EH, Royster LH, Thomas WG. Presumed noise-induced permanent threshold shift resulting from exposure to an A-weighted Leq of $89 \mathrm{~dB}$. Journal of the Acoustical Society of America 1978; 64: 192-7.

18. Niskar AS, Kieszak SM, Holmes AE, Esteban E, Rubin C, Brody DJ. Estimated Prevalence of Noise-
Induced Hearing Threshold Shifts Among Children 6 to 19 Years of Age: The Third National Health and Nutrition Examination Survey, 1988-1994, United States. Pediatrics 2001; 108: 40-3.

19. Widen SE, Bohlin M, Johansson I. Gender perspectives in psychometrics related to leisure time noise exposure and use of hearing protection. Noise and Health 2011; 13: 407-14.

20. Smith P, Davis A, Ferguson M, Lutman M. The prevalence and type of social noise exposure in young adults. Noise and Health 2000; 2: 41-56.

21. Henderson D, Subramaniam M, Boettcher FA. Individual susceptibility to noise induced hearing loss: an old topic revisited. Ear Hear 1993; 14: 152-68.

22. Lu J, Cheng X, LI Y, Zeng L, Zhao Y. Evaluation of individual susceptibility to noise-induced hearing loss in textile workers in China. Arch of Environ Occup Health 2005; 60: 287-94.

23. Sliwinska-Kowalska M, Noben-Trauth K, Pawelczyk M, KowaLSKI TJ. Single nucleotide polymorphisms in the Cadherin 23 (CDH23) gene in Polish workers exposed to industrial noise. Am J of Hum Biol 2008; 20: 481-3.

24. Van Laer L, Carlsson PI, Ottschytsch N, y col. The contribution of genes involved in potassiumrecycling in the inner ear to noise-induced hearing loss. Hum Mutat 2006; 27: 786-95.

25. Pawlaczyk-Luszczyniska M, Dudarewicz A, Waszkowska M, SzYMczaK W, SLIWINSKa-Kowalska M. The impact of low-frequency noise on human mental performance. Int J Occup Med Environ Health 2005; 18: 185-98.

26. Gates GA, Schmid P, Kujawa SG, Nam B, D’Agostino R. Longitudinal threshold changes in older men with audiometric notches. Hear Res 2000; 141: 220-8.

27. ROSENHALL U. The influence of ageing on noiseinduced hearing loss. Noise and Health 2003; 5 : 47-53.

28. SCHMIEDT RA. The Physiology of Cochlear Presbycusis. En: Gordon-Salant S y cols, eds, The Aging Auditory System. New York: Springer 2010; 9-38.

29. Kujawa SG, Liberman MC. Acceleration of AgeRelated Hearing Loss by Early Noise Exposure: Evidence of a Misspent Youth. The Journal of Neuroscience 2006; 26: 2115-23.

Dirección: Dr. Eduardo Fuentes L.

Escuela de Fonoaudiología, Universidad San Sebastián

E mail: eduardo.fuentes@uss.cl 